\title{
Simultaneous associated onset of atrial fibrillation and ventricular tachycardia without structural cardiopathy - A
} case report

Eduardo Esteban-Zubero*, Cristina García-Muro, Moisés Alejandro Alatorre-Jiménez, Alejandro Marín-Medina, Carlos Arturo LópezGarcía, Ahmed Youssef and Rocío Villeda-González

Emergency Department, Hospital San Pedro, Logroño, Spain

\begin{abstract}
Onset of fast ventricular tachycardia after spontaneous termination of atrial fibrillation is unusual and the mechanism is not well known. There are few case reports in the literatura and the authors did not found the physiopathological points of this entity. Hereby we present a 73-year-old man atrial fibrillation with short periods of non-sustained ventricular tachycardia treated with amiodarone effectively. Coronary angiography, an ergometry as well as an echocardiogram were realized to complete the study showing any disturbs.
\end{abstract}

\section{Case report}

A 73-year-old man with a history of hypertension, and obstructive sleep apnea syndrome was admitted in our emergency department due to oppresive chest pain and general discomfort. He denied loss of consciousness after the event or other symptoms. At home, an electrocardiogram was performed revealing short periods of nonsustained ventricular tachycardia (NSVT). Due to that, it was started treatment with amiodarone (300 $\mathrm{mg}$ endovenous in bolus).

On physical examination, it was revealed arrhythmic heart tones without pulmonary or abdominal disturbs. A new electrocardiogram was performed revealing an unknown atrial fibrillation (AF) with short peridos of NSVT (Figure 1). To complete the study, a blood test was performed revealing any disturbs (including ultra-sensitive cardiac tronponins). A chest $\mathrm{x}$-ray was also realized showing any disturbs. Attending to these results, amiodarone treatment $(600 \mathrm{mg}$ endovenous in $250 \mathrm{cc}$ of glucose $5 \%$ infusion, $21 \mathrm{ml} / \mathrm{h}$ ) was initiated decreasing VT episodes (Figure 2). The patient was transferred to the intensive care unit for control during $24 \mathrm{~h}$ discharging after that to Cardiology unit with an electrocardiogram in sinusal rhytm with left anterior fascicular block and first grade block (like previously) (Figure 3). During this process, a coronary angiography, an ergometry as well as an echocardiogram were realized showing any disturbs. After two weeks of cardiac monitoring without new episodes of VT or AF, the patient was discharged.

\section{Discussion}

$\mathrm{AF}$ is an entity characterized by rapid and irregular RR intervals. Onset of fast VT after spontaneous termination of AF is unusual and the mechanism is not well known [1]. Idiopathic left VT is probably based on a re-entrant mechanism, and right ventricular outflow tract tachycardia could be due to triggered activity or abnormal automaticity [2]. To explain VT periods during AF, authors have been suggested different theories. A long-short RR interval sequence during AF could create unidirectional block and start re-entry. Long preceding RR intervals have been noted in $77 \%$ of cases with "repetitive monomorphic VT and a structurally normal heart" [3]. This theory may be the cause of our case report due to the abscence of structural heart diseases. On the other hand, it has been observed that irregular RR intervals during AF could lead to rapid escalation of the pause dependent after depolarisations above a critical threshold initiating VT. In addition, overdrive excitation during AF could lead to $\mathrm{Ca} 2+$ overloading and abnormal automaticity [2].

This rare entity of AF with onset of VT has been related with some diseases, including Left ventricular noncompaction and Brugada syndrome [4], hypertrophic cardiomyopathy [5], and myotonic dystrophy [6]. To improve the knowledge of this unusual pathology, more studies are required in the future to explain the physiopathological aspects. Until that momento, avoiding the episodes of AF may reduce the number of VT episodes, particularly, in patients with severe left ventricular impairment.

${ }^{\star}$ Correspondence to: Eduardo Esteban-Zubero, Department of Emergency, Hospital San Pedro, Logroño, Spain, E-mail: eezubero@gmail.com

Key words: atrial fibrillation, ventricular tachycardia, cardiopathy

Received: April 02, 2019; Accepted: April 19, 2019; Published: April 22, 2019 


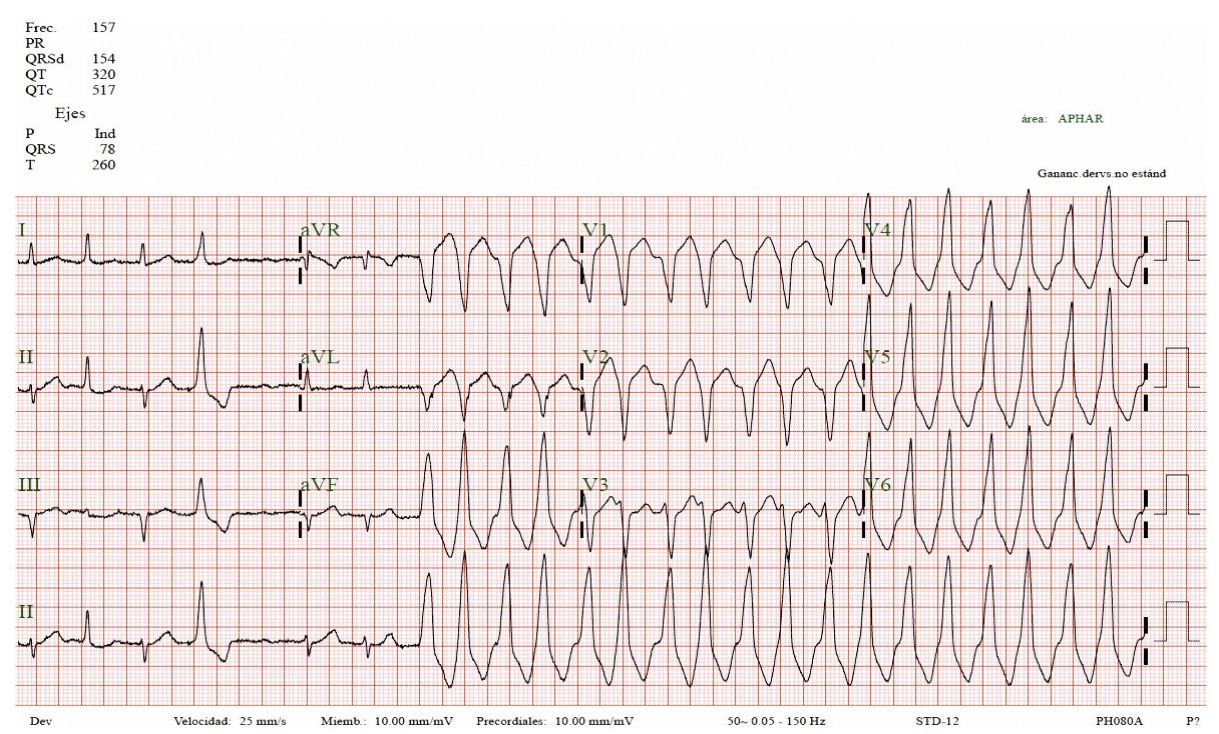

Figure 1. Electrocardiogram revealing an unknown atrial fibrillation with short periods of non-sustained ventricular tachycardia

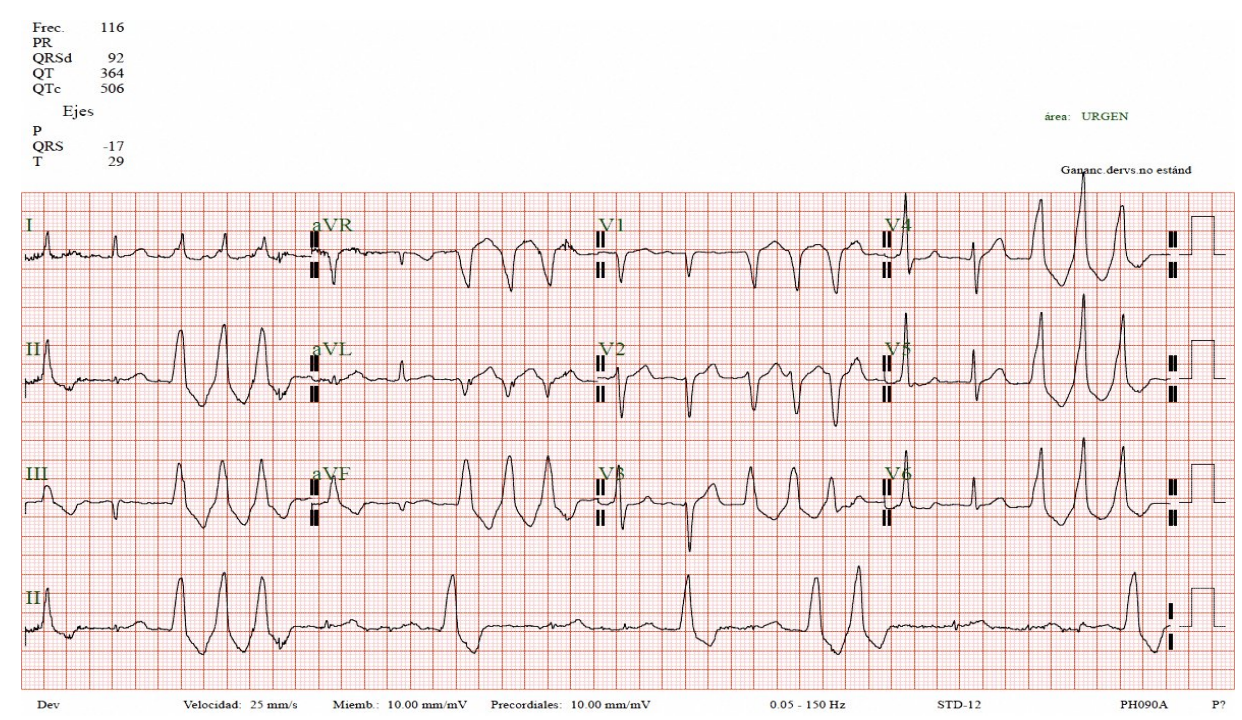

Figure 2. Electrocardiogram after the beginning of amiodarone treatment revealing atrial fibrillation with short periods of non-sustained ventricular tachycardia. A decrease of ventricular tachycardia episodes may be observed

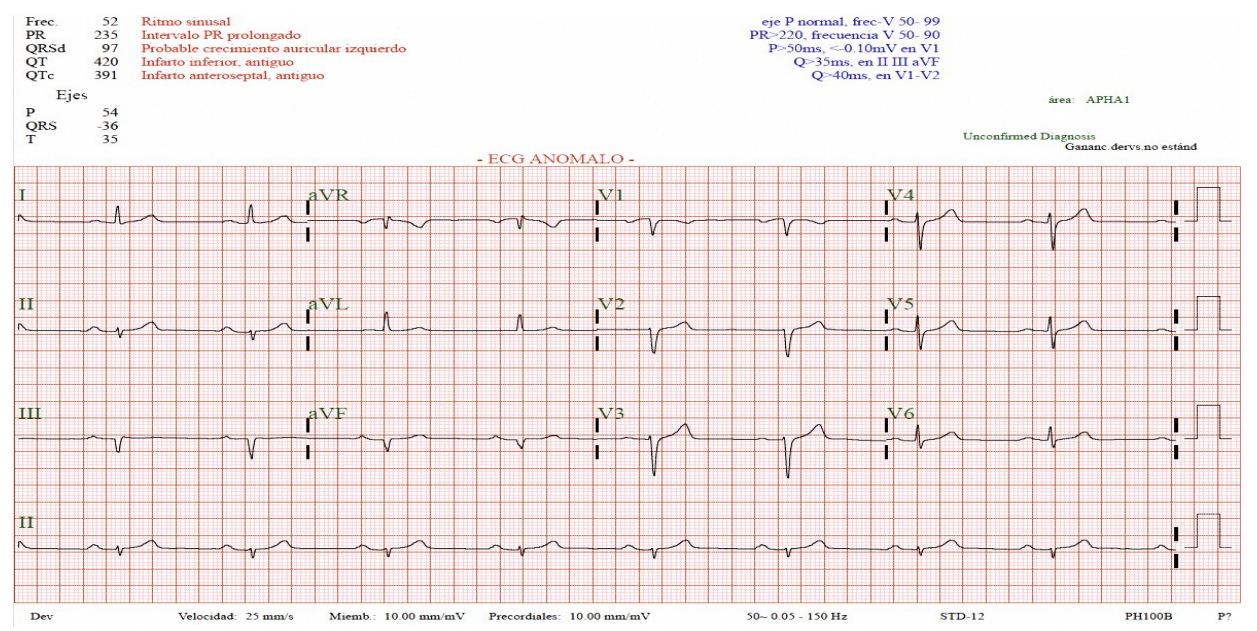

Figure 3. Electrocardiogram in sinusal rhytm with left anterior fascicular block and first grade block 


\section{References}

1. Osman F, Ward R, Morley-Davies A (2008) Simultaneous spontaneous onset of ventricular tachycardia and termination of atrial fibrillation. Europace 1: 171-172.

2. Nabar A, Rodriguez LM, Timmermans C, Kattenbeck K, Wellens HJ (2000) Capture and fusion beats during atrial fibrillation and ventricular tachycardia. Heart 84: E1.

3. Zimmermann M, Maisonblanche P, Cauchemez B, Leclercq JF, Coumel P (1986) Determinants of the spontaneous ectopic activity in repetitive monomorphic idiopathic ventricular tachycardia. J Am Coll Cardiol 7: 1219-1227.
4. Kaźmierczak J, Zielonka J, Peregud-Pogorzelska M, Kiedrowicz R, Wielusiński M (2011) Ventricular and supraventricular arrhythmias and heart failure in a patient with left ventricular noncompaction and Brugada syndrome. Cardiol J 18: 310-313.

5. Boriani G, Rapezzi C, Biffi M, Branzi A, Spirito P (2002) Atrial fibrillation precipitating sustained ventricular tachycardia in hypertrophic cardiomyopathy. J Cardiovasc Electrophysiol 13: 954.

6. Eroğlu S, Ozin B, Ozbiçer S, Müderrisoğlu H (2009) A case of myotonic dystrophy presenting with ventricular tachycardia and atrial fibrillation. Turk Kardiyol Dern Ars 37: 337-340.

Copyright: (C2019 Esteban-Zubero E. This is an open-access article distributed under the terms of the Creative Commons Attribution License, which permits unrestricted use, distribution, and reproduction in any medium, provided the original author and source are credited. 